\title{
Journalism, Misinformation and Fact Checking Chairs' Welcome
}

It is our pleasure to welcome you to the WWW 2018 Journalism, Misinformation and Fact Checking Alternate Track. Although the problem of misinformation and deceptive information is as old as Web itself, the topic has gained a lot of attention recently. Phenomena, such as misinformation propagation, fabricated news reports (also known as "fake news",) computational propaganda, astroturf, and ideological polarization have become more common on the Web and the social Web, calling for a cross-cutting approach to better understand the topic. One approach that has gained some traction is that of the establishment of fact-checking organizations.

This track solicited contributions that explore the range of computational, social, cognitive, economic, and communication topics related to the above phenomena. We received submissions covering a broad range of topics, including computational approaches for detecting misinformation and propaganda on the Web and social media, as well as proposals to improve fact checking, critical thinking, information and media literacy, crowdsourcing, and societal decision-making processes.

The call attracted submissions from North America, Europe, Asia, and Africa. The program committee selected contributions for oral presentation. Review and acceptance statistics are as follows:

\begin{tabular}{|l|c|c|c|}
\hline Submission Type & Reviewed & Accepted & $\%$ Acc. \\
\hline Full Technical Papers & 43 & 12 & $28 \%$ \\
\hline
\end{tabular}

Giovanni Luca Ciampaglia

Co-Chair

Indiana University Network Science Institute

Kristina Lerman

Panagiotis Metaxas

Co-Chair

Co-Chair

USC Information Sciences Wellesley College

Institute

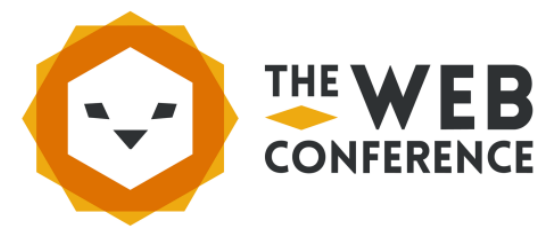




\section{Journalism, Misinformation and Fact Checking Organization}

Workshop/Track Chairs: Giovanni Luca Ciampaglia (Indiana University, USA)

Kristina Lerman (University of Southern California, USA)

Panagiotis Metaxas (Wellesley College, USA)

Program Committee: Harith Alani (The Open University, United Kingdom)

Jisun An (Hamad Bin Khalifa University, Qatar)

Matt Baum (Harvard University, USA)

Guido Caldarelli (National Research Council, Italy)

Carlos Castillo (Eurecat, Spain)

James Caverlee (Texas A\&M University, USA)

Meeyoung Cha (KAIST, South Korea)

Robin Cohen (University of Waterloo, Canada)

Nicholas Diakopoulos (Northwestern University, USA)

Lucia Falzon (Defence Science and Technology Group, Australia)

Emilio Ferrara (University of Southern California, USA)

Aram Galstyan (University of Southern California, USA)

Kelly Garrett (Ohio State University, USA)

Amira Ghenai (University of Waterloo, Canada)

Yevgeniy Golovchenko (University of Copenhagen, Denmark)

Nir Grinberg (Northeastern University, USA)

Noriko Hara (Indiana University, USA)

Naeemul Hassan (University of Mississippi, USA)

Jim Hendler (Rensselaer Polytechnic Institute, USA)

Jeff Jarvis (CUNY, USA)

Prakruthi Karuna (George Mason University, USA)

Brian Keegan (University of Colorado Boulder, USA)

Johannes Kiesel (Bauhaus-Universität Weimar, Germany)

Hemank Lamba (Carnegie Mellon University, USA)

Gerry Lanosga (Indiana University, USA)

Dongwon Lee (Penn State University, USA)

Xiao Ma (Cornell Tech, USA)

Alexios Mantzarlis (International Fact-Checking Network, USA)

Winter Mason (Facebook, Inc., USA)

Gregory Maus (Indiana University, USA)

Miriam Metzger (UC Santa Barbara, USA)

An Mina (Harvard University, USA)

Tanushree Mitra (Virginia Tech, USA)

Elaheh Momeni (University of Vienna, USA)

Fred Morstatter (University of Southern California, USA)

Eni Mustafaraj (Wellesley College, USA) 
Program Committee (continued): Christine Ogan (Indiana University, USA)

John Paolillo (Indiana University, USA)

David Rothschild (Microsoft Research, USA)

Giancarlo Ruffo (University of Turin, Italy)

Nikos Sarris (Athens Technology Center, Greece)

Kazutoshi Sasahara (Nagoya University, Japan)

Nishanth Sastry (King's College London, United Kingdom)

Craig Silverman (Buzzfeed, Canada)

Emmanuel Vincent (Climate Feedback, USA)

Tim Weninger (University of Notre Dame, USA)

Christo Wilson (Northeastern University, USA)

Jun Yang (Duke University, USA)

Cong Yu (Google Research, USA)

Amy Zhang (Massachusetts Institute of Technology, USA)

Arkaitz Zubiaga (Warwick University, United Kingdom) 